\title{
BODY WEIGHT AS A DETERMINANT OF CLINICAL EVOLUTION IN HAMSTERS (Mesocricetus auratus) INFECTED WITH Leishmania (Viannia) panamensis
}

\author{
Ángela María GÓMEZ-GALINDO \& Lucy Gabriela DELGADO-MURCIA
}

\begin{abstract}
SUMMARY
The clinical outcome of infection with Leishmania species of the subgenus Viannia in hamster model (Mesocricetus auratus) has shown to be different depending on experimental protocol. Body weight has been a relevant determinant of the clinical outcome of the infection in hamsters with visceral leishmaniasis but its importance as a clinical parameter in hamsters with cutaneous leishmaniasis is not known. In this study, the clinical evolution of infection with $L$. (V) panamensis was evaluated in juvenile and adult male hamsters during 11 weeks by comparing clinical parameters such as attitude, temperature, respiratory rate, appearance of the stool, and body weight between infected and non-infected groups. Results showed that body weight decreased in adult hamsters after infection by $L$. (V) panamensis; this observation supports the use of body weight as an additional parameter to define the management or treatment of cutaneous leishmaniasis in infected adult hamsters used as an animal experimental model for leishmaniasis.
\end{abstract}

KEYWORDS: Leishmaniasis; Hamsters infection; Body weight.

\section{INTRODUCTION}

Leishmaniasis is a zoonotic parasitic disease caused by protozoa from the genus Leishmania, for which different mammals have been identified as potential reservoirs and susceptible host ${ }^{18}$. Globally, about 350 million people in over 98 countries live at risk of infection with Leishmania parasites. The disease is classified into three forms depending on its clinical presentation: cutaneous, mucocutaneous and visceral leishmaniasis, of which the most prevalent one is cutaneous leishmaniasis ${ }^{19}$.

In Colombia, about nearly $99 \%$ of cases present as cutaneous leishmaniasis and a large proportion of these cases (70\%) are caused by Leishmania (Viannia) panamensis $[\text { L. (V) panamensis }]^{16}$.

Progress in the development of new prophylactic and therapeutic strategies to control leishmaniasis depends largely on the characterization of animal models that accurately reproduce the course of the infection in human to allow a complete understanding of the infection and its evolution ${ }^{3}$.

Hamsters are considered a suitable animal model for infection with Leishmania parasites of the subgenus Viannia (New World parasites) because of their special susceptibility to the infection with these species and because the chronic progression of the disease is similar to the one developed in humans that are naturally infected with Leishmania ${ }^{3,11,12,13}$.
Particularly for this animal model, the clinical outcome of infection with Leishmania species of the subgenus Viannia, including $L$. $(V)$ panamensis, has been shown to have different characteristics, and these differences have been shown to be related to biological aspects such as age and gender. It has been demonstrated that male hamsters infected at a juvenile stage are less susceptible to the infection than adult male hamsters ${ }^{14}$. Also, body weight has been shown to be a relevant determinant of the clinical outcome of the infection in hamsters with visceral leishmaniasis ${ }^{4}$, but its importance as a clinical parameter in hamsters with cutaneous leishmaniasis is not known.

Based on all these observations, we evaluated some clinical parameters such as attitude, temperature, respiratory rate, appearance of the stool, and body weight in juvenile and adult male golden hamsters (Mesocricetus auratus) intradermally infected with $L$. $(V)$ panamensis to determine the relationship of these biological parameters with clinical evolution of cutaneous leishmaniasis.

\section{MATERIALS AND METHODS}

1. Animals: The study group consisted of 20 male golden Syrian hamsters (Mesocricetus auratus) obtained from the breeding facility of the Colombian National Institute of Health (Bogotá, Colombia). Hamsters were housed in the animal facility of the Department of Pharmacy at the National University of Colombia where they had a quarantine period of two weeks before being infected. 


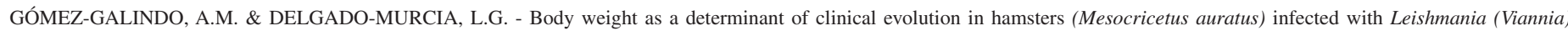
panamensis. Rev. Inst. Med. Trop. Sao Paulo, 55(5): 357-62, 2013.

Hamsters were kept in individual transparent polycarbonate boxes with wood shawing bedding and ad libitum access to fresh water and Rodentina ${ }^{\circledR}$ (Purina, Co, Mosquera, Cundinamarca).

2. Study groups: Hamsters were divided according to age and weight into two study groups. The first group consisted of ten juvenile hamsters (3-4 weeks old) and the second group of ten adult hamsters (7-8 weeks old). Three hamsters from each group were left uninfected as controls.

3. Parasites: Parasites used in this study were metacyclic promastigotes from the L. (V) panamensis strain (MHOM/CO/87/ UA140), which were kindly provided by Dr. Sara Robledo from the University of Antioquia (Medellin, Colombia). Parasites were grown at $27^{\circ} \mathrm{C}$ under room humidity and atmospheric conditions in $25 \mathrm{~cm}^{2}$ culture dishes (Techno Plastic Products AG, Switzerland) with RPMI and 5\% fetal bovine serum (FBS) supplemented with 1\% L-glutamine. Parasites were maintained with 5-6 mL of culture medium for seven days. After this period, parasites were placed in $15 \mathrm{~mL}$ tubes and centrifuged at 1,500 rpm. Promastigotes were collected at the stationary phase of growth for the subsequent infection assays. To calculate the number of parasites for the infection assays, a $50-\mu \mathrm{L}$ aliquot of parasite culture was mixed with $50 \mu \mathrm{L}$ of saline solution containing $2 \%$ Giemsa and $2 \%$ formaldehyde in order to immobilize parasites and facilitate their counting. The evaluation of parasites was performed in a Neubauer chamber.

4. Inoculation of hamsters: Hamsters were inoculated under anesthesia by intramuscular administration of ketamine (Imalgene $\left.1000^{\circledR}\right)$ and xylazine $\left(2 \%\right.$ Rompun $\left.^{\circledR}\right)$ at the recommended doses: ketamine $(100 \mathrm{mg} / \mathrm{kg})$ and xylazine $(10 \mathrm{mg} / \mathrm{kg})^{5}$. After removing the hair from the inoculation area, parasites were inoculated intradermally at the lumbosacral area using a 1-mL syringe. Juvenile and adult hamsters were infected with $1 \times 10^{6} \mathrm{~L}$. (V) panamensis promastigotes in $200 \mu \mathrm{L}$ of sterile physiological saline solution (PSS). The control hamsters were injected intradermally with $200 \mu \mathrm{L}$ of sterile solution PSS.

5. Clinical evaluation: After infection with $L$. (V) panamensis promastigotes, adults and juvenile hamsters were monitored for eleven weeks. Data on the initial body weight of the animals were gathered on the day of infection (Day 0) using a calibrated balance (Sartorius ${ }^{\circledR}$ ), which has an error factor of $\pm 0.01 \mathrm{~g}$. Weight measures were taken postinfection four times a week, monitoring also other aspects related to the animals health such as attitude, condition of the mucous membranes (color and moisture), respiratory rate, rectal temperature and aspect of the stool ${ }^{15}$. The evolution of lesions (inoculated area from papule to open ulcer) were also checked and measured with a Vernier Calliper, which allowed determining the area of lesions (Transverse diameter of the lesion $\mathrm{X}$ Sagittal diameter of the lesion).

On week 11 hamsters were sacrificed according to the Guidelines on Euthanasia of the American Veterinary Medical Association ${ }^{1}$. Briefly, sodium pentobarbital was intravenously administered to sacrificed animals at a dosage of $100 \mathrm{mg} / \mathrm{kg}$ of body weight. Aspirates of cutaneous lesions and imprints of blood and visceral organs were then collected to determine whether parasites were present at the site of the lesion and/or parasites had migrated causing possible metastasis. In brief, aspirates were collected using a 1-mL syringe after cleaning the skin of the lumbosacral area (if there was no injury, the aspirate was collected at the site of inoculation). Each aspirate was spread on a glass slide, and imprints too left to air dry, then added $100 \%$ methanol, left to air dry for five minutes, then stained with $20 \%$ Giemsa (Chemi Reagents Ltda., Bogotá, Colombia). After 25 minutes, each sheet was washed with Giemsa Buffer $\mathrm{pH} 7.4$ and examined under a light microscope (Fig. 1).

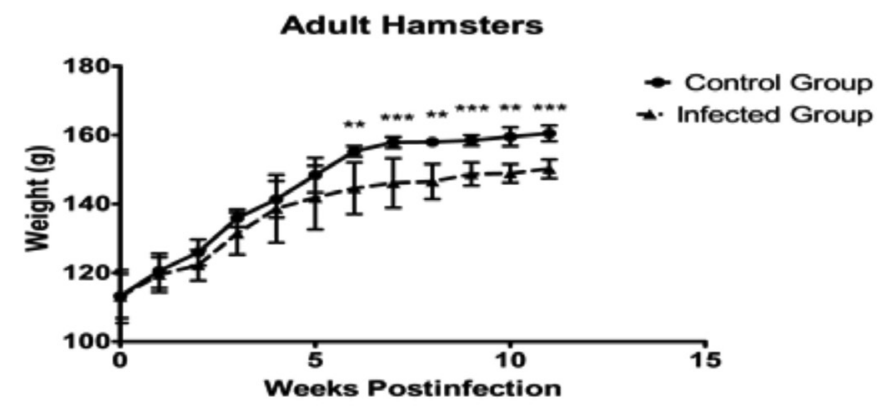

Fig. 1 - Changes in the weight of adult hamsters (seven to eight weeks of age) infected with $1 \times 10^{6} \mathrm{~L}$. (V) panamensis parasites. The $x$-axis corresponds to the weeks of study, while the $y$-axis shows the weights of the hamsters starting at the moment of infection or time zero (0). Weight gain was monitored until Week 11 post infection (19 weeks of age). Data represents mean of weight $( \pm \mathrm{SEM}), p$-values of $\leq 0.05$ were considered significant. $* * * p<0.0001$, $* * p<0.005$.

6. Experimental design and statistical analysis: Clinical parameters such as the respiratory rate and rectal temperature between infected and non-infected animals were compared by performing a Student's $t$-test, considering $p$-values $\leq 0.05$ to be statistically significant. Data were plotted as mean \pm SEM using the GraphPad Prism 5 software. Other clinical parameters evaluated such as attitude, condition of the mucus membranes (color and moisture), and the aspect of the stool were assigned a numerical value between 1 and 3 , where 2 is considered a normal value, and the differences between these parameters were evaluated with at-test. The number of animals in the study was selected according to one of three principles of the $3 R^{\prime}$ 's for the reduction in the number of animals in experiments (using the software "Java applets for power and sample size", with an alpha of 0.05).

7. Ethical considerations: All assays were performed in accordance to the provisions provided in the Colombian law 84 of 1989 (National Statute for the Protection of Animals) and under Title V of Resolution 008430 of 1993 emitted by the Ministry of Health about biomedical research involving animals ${ }^{2,10}$. Housing and space conditions followed in this study adhered to the Guide for the Care and Use of Laboratory Animals of the National Research Council ${ }^{6}$. This assays included in the magister project were approved by The Ethics Committee of Science Faculty (Universidad Nacional de Colombia) at April 11 of 2011.

\section{RESULTS}

1. Clinical evaluation: The clinical parameters evaluated in this study remained constant in the hamsters infected with $L .(V)$ panamensis. The respiratory rate remained within the normal range reported for hamsters (30-140 breaths/minute), the rectal temperature ranged between $38.1 \pm 1.3{ }^{\circ} \mathrm{C}$, which is considered a normal value, while the animal's attitude, condition of the membranes (color and moisture), and the aspect of the stool were within the normal ranges for juvenile and adult animals ${ }^{15}$. 
There was no skin ulcers in juvenile hamsters infected with $L .(V)$ panamensis, although papules without presenting ulceration (Fig. 2) were observed in all individuals from week five post-infection in the adult group (one week before the detection of the significant variation of weight) until the final day of the observation. It is important to clarify that papules appeared only during the fifth week (none after this period). The average lesions size was $8 \mathrm{~mm}$ in transverse and sagittal diameter, which were then shown to contain parasites when the aspirate was examined (Fig. 1).

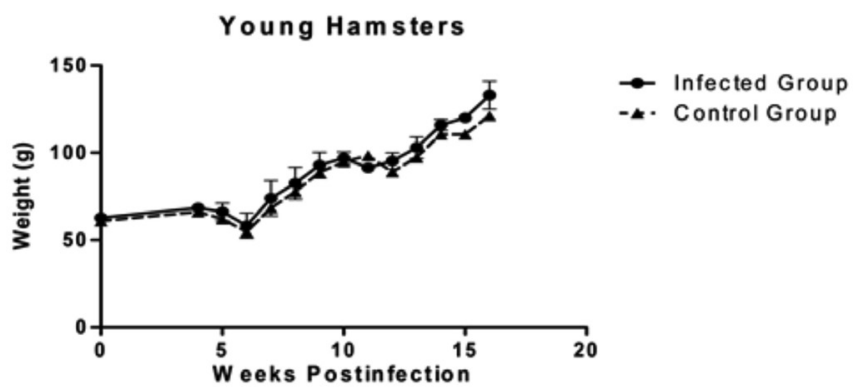

Fig. 2 - Changes in the weight of juvenile hamsters (three to four weeks of age) infected with $1 \times 10^{6} \mathrm{~L}$. (V) panamensis parasites. The $\mathrm{x}$-axis corresponds to the weeks of study while the $y$-axis shows the weights of the hamsters starting at the moment of infection or time zero $(0)$. Weight gain was monitored until week 16 post-infection (20 weeks of age). Data represent mean of weight $( \pm \mathrm{SEM}), p$-values of $\leq 0.05$ were considered significant.

Five adult infected hamsters presented metastasis of parasites to other tissues, when smears were made of visceral organs at sacrifice; particularly in the liver.

2. Weight: There was no statistically significant difference between the weights of juvenile hamsters infected with $L$. (V) panamensis in relation to the control hamsters until the last week of the study (week 11).

Starting on week 6 post-infection there was a significant variation in the weight of the adult hamsters, which remained significant until the last week of the study.

The weight of the adult hamsters varied significantly from week 6 post-infection until the end of the study, compared to the control group (approximately $12 \mathrm{~g}$ ), as shown in Figures 3 and 4 where it can be observed that the control group gained weight whereas the gained weight of the infected hamsters stopped throughout the study.

\section{DISCUSSION}

From the sixth week, the weight between the group of infected hamsters and the control group without infection was significantly different ( $p$ values $\leq 0.05$ ). Additionally, body weight can be an early indicator of the disease process, even before the appearance of ulcers.

It has been reported that progression of leishmaniasis infections are often associated with testosterone levels in males, therefore, hamsters that are infected at youth tend to show a slower disease progression ${ }^{14}$, while those infected at adulthood show a more rapid progression of the disease. This was consistently observed in our study and was correlated with variations in animal's body weight.

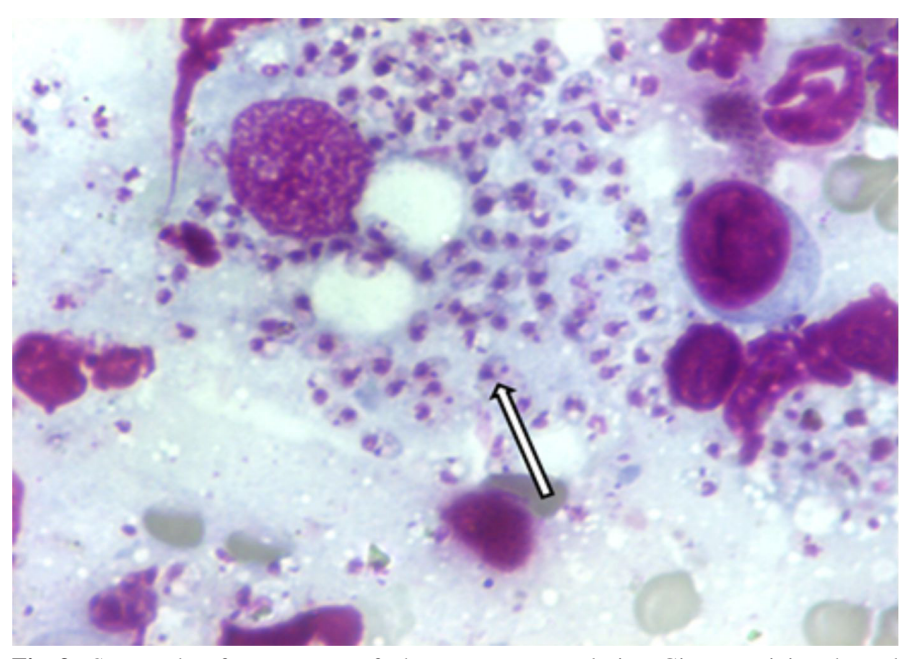

Fig. 3 - Smear taken from contents of a hamster cutaneous lesion. Giemsa staining showed a mononuclear cell with two nuclear structures in its cytoplasm (Amastigotes). This finding is associated with the infection with $L$. (V) panamensis.

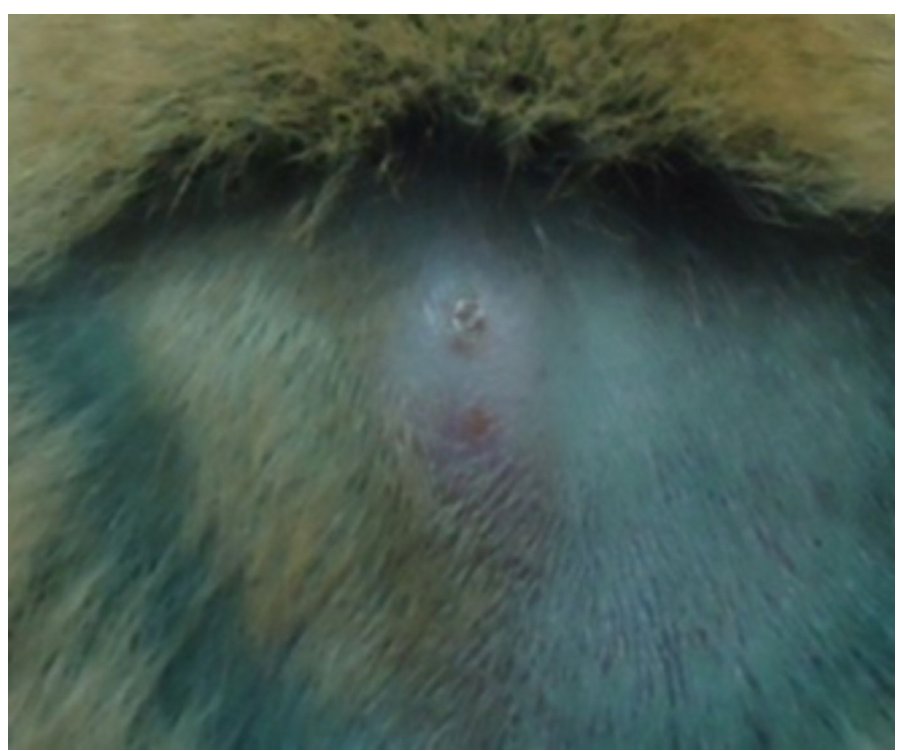

Fig. 4 - Lesion caused by L. (V) panamensis in dorsal skin of an adult hamster. The lesion was presented at week 5 post-infection, with a transverse diameter of $6 \mathrm{~mm}$ and sagittal diameter of $8 \mathrm{~mm}$. This lesion never ulcerated.

WYLLIE \& FAIRLAMB $(2006)^{20}$ consider body weight to be a good indicator of infection in early stages of visceral leishmaniasis since hamsters infected with Leishmania donovani tend to gain weight more slowly than non-infected hamsters. Similar results were found in this study, as we found significant differences in weight gain between control hamsters and the ones infected with $L$. $(V)$ panamensis that developed cutaneous symptoms. The current is the first report related to clinical outcomes in experimental hamster infection with $L$. $(V)$ panamensis. Here, it is important to remember that this Leishmania species do not induce the visceralizing disease due to their cutaneous tropism, besides the number of parasites inoculated ( $1 \times 10^{6}$ promastigotes de $L$. $(\mathrm{V})$ panamensis) has not been described as responsible for visceralizing process ${ }^{17}$. In our work, parasite detection in liver imprints was not necessarily associated with 


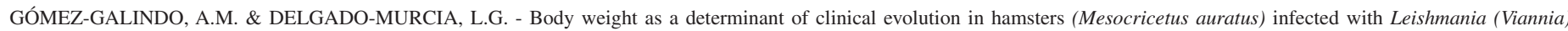
panamensis. Rev. Inst. Med. Trop. Sao Paulo, 55(5): 357-62, 2013.

their liver colonization because we took imprints on a surface of liver rich in blood, a fluid which could be responsible for providing these parasites in the sample. The suggestion is based on the observation of parasite structures into macrophages derived from blood (by cardiac puncture). Additionally, no animal showed hepatosplenomegaly, a critical clinical point observed in cases of visceral leishmaniasis ${ }^{17}$.

It is important to emphasize that two of the infected animals in adult age also showed a significantly low weight with respect to animals from the control group and they did not show parasite structures in imprints (blood or visceral organs); for these reasons, we did not relate the weight reduction with an infection to visceral level, we associated this characteristic with a chronic cutaneous process and/or with the cytokines production (as tumor necrosis factor or transforming growth factor- $\beta)^{9}$.

It has been reported in hamsters infected with Leishmania (Viannia) guyanensis that cachexia has been shown to be an early manifestation of dissemination throughout the lymphoid organs, and secondary cutaneous lesions ${ }^{8}$. We did not detect secondary post-infection injuries in skin (without parasites in lymph nodes or spleen), therefore we could not affirm that our findings (weight loss) represent an early manifestation of dissemination to other organs (liver or spleen); it is more probable that they represent the blood parasite circulation previous to their dissemination to another layer of the skin. For this reason, we consider that weight could be an indirect parameter (plus some cytokines) of the clinical state and chronicity of the infection by L. (V) panamensis.

Although skin lesions are mostly a compartmentalized phenomenon (local), two situations cannot be ruled out to contribute to the possible systemic dissemination of the parasite and favor visceral colonization in the vertebrate host. This situation had happened in five animals infected at adult age. First, that this type of parasite may disseminate to the internal organs in susceptible individuals, and/or second, factors associated with parasite, such as virulence. We did not find in our work, evidence that related the weight reduction to a visceral infection.

Determining the body weight may be useful as an additional indicator when monitoring adult hamsters infected with $L$. $(V)$ panamensis prior to the onset of skin lesions and dissemination of parasites to other skin's sites or metastatic to visceral organs.

Studies in animal models have helped to determine some features of the clinical and pathological evolution of multiple infectious agents, including leishmaniasis parasites. They have also help designing new therapeutic approaches to the disease by using these living organisms that resemble to some extent the possible responses that occur in infected humans.

It is therefore important to determine patterns of infection in order to establish a methodology for use of the hamster model as a tool for studying leishmaniasis, the weight in $L$. $(V)$ panamensis cutaneous infections had not been previously reported as an indicator of the infection process (less in localized skin process).

OSORIO et al., $(1998)^{11}$ pointed to the hamster model as an excellent tool for the study of leishmaniasis caused by parasites belonging to the subgenus Viannia due to it having shown susceptibility to these parasites and the similarity of the clinical symptoms to the ones presented in humans [in particular in relation to the presentation of chronic recurrent ulcers]. However in this study, no ulcerative lesions developed in juvenile hamsters by inoculation of $1 \times 10^{6} \mathrm{~L}$. (V) panamensis promastigotes, while adult hamsters showed papules that contained parasites inside. The weight in hamsters infected with human cutaneous Leishmania can be used as an additional parameter to help define the success of new therapeutic alternatives for this disease.

\section{RESUMO}

\section{Peso como determinante da evolução clínica em hamsters (Mesocricetus auratus) infectados com Leishmania (Viannia) panamensis}

O resultado clínico da infecção por espécies de Leishmania do subgênero Viannia no modelo de hamster (Mesocricetus auratus) tem se mostrado diferente, dependendo do protocolo experimental. $\mathrm{O}$ peso corporal tem sido um importante determinante da evolução clínica da infecção em hamsters com leishmaniose visceral, mas sua importância como parâmetro clínico em hamsters com leishmaniose cutânea não é conhecido. Neste estudo, a evolução clínica da infecção com L. (V) panamensis foi avaliada em jovens e adultos hamsters machos durante 11 semanas, comparando os parâmetros clínicos tais como a atitude, a temperatura, a frequência respiratória, a aparência das fezes, e o peso corporal entre infectado e grupos não infectados. Os resultados mostraram que o peso corporal diminuiu em hamsters adultos após infecção por $L$. (V) panamensis. Esta observação suporta a utilização do peso corporal, como um parâmetro adicional para definir a administração ou o tratamento de leishmaniose cutânea em hamsters adultos infectados usados como modelo animal experimental para a leishmaniose.

\section{ACKNOWLEDGEMENTS}

We would like to express our sincere gratitude to all members of the Immunotoxicology Research Group and to Professor Luis Fernando Ospina Giraldo, Head of Biotery of the Department of Pharmacy, Universidad Nacional de Colombia.

\section{FUNDING}

This study was performed with resources obtained from the Dirección de Investigación Sede Bogotá (DIB, project No. 16015), the Universidad Nacional de Colombia (project No. 15098) and the Departamento de Ciencia Tecnología e Innovación-Colciencias through project 110151928476 . These organizations sponsor research, but have no say in the design, analysis, interpretation and decision to submit the paper for publication.

\section{AUTHOR CONTRIBUTIONS}

Ángela María Gómez Galindo (as Magister student of Pharmaceutical Sciences Program) realized all experimental procedures, obtaining and analyzing the corresponding results. Gabriela Delgado, as head of the Immunotoxicology Research Group, directed the work. She also analyzed results and oriented the focus of this manuscript. Both of them wrote this text. 


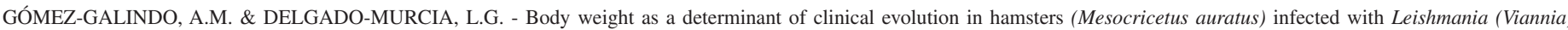
panamensis. Rev. Inst. Med. Trop. Sao Paulo, 55(5): 357-62, 2013.

\section{REFERENCES}

1. American Veterinary Medical Association. Guidelines on Euthanasia, Formerly Report of the AVMA Panel on Euthanasia. Noninhalant Pharmaceutical Agents. Barbituric Acid Derivatives. Schaumburg: AVMA; 2007. p. 11-12.

2. Congreso de la República de Colombia. Por la cual se adopta el Estatuto Nacional de Protección de los Animales y se crean unas contravenciones y se regula lo referente a su procedimiento y competencia. 1989;1-9.

3. De Oliveira CI, Teixeira MJ, Gomes R, Barral A, Brodskyn C. Animal models for infectious diseases caused by parasites: leishmaniasis. Drug Discovery Today. 2004; 1: 81-86.

4. Evans TG, Smith D, Pearson RD. Humoral factors and nonspecific immune suppression in Syrian hamsters infected with Leishmania donovani. J Parasitol. 1990;76:212-7.

5. Flecknell P. Laboratory animal anesthesia: a practical introduction for research workers and technicians. $2^{\text {nd }}$ ed. London: Academic Press; 1996. p. 172-4.

6. Garber J, Wayne B, Bielitzki JT, Clayton LA, Donovan JC, Hendriksen CF, et al. Institute of Laboratory Animal Research, Committee for the Update of the Guide for the Care and Use of Laboratory Animals National Research Council. Guide for the care and use of laboratory animals. $8^{\text {th }}$ ed. Washington: The National Academies Press; 2001. p. 21-202.

7. Lenth RV. Java Applets for Power and Sample Size [Computer software]. [cited 2012 Aug 8]. Available from: http://www.stat.uiowa.edu/ rlenth/Power.

8. Martínez JE, Valderrama L, Gama V, Leiby DA, Saravia, NG. Clonal diversity in the expression and stability of the metastatic capability of Leishmania guyanensis in the golden hamster. J Parasitol. 2000;86:792-9.

9. Melby P, Andrade F, Darnell B, Valencia G, Tryon V, Palomo A. Increased expression of proinflammatory cytokines in chronic lesions of human cutaneous leishmaniasis. Infect Immun. 1994;62:837-42.

10. Ministerio de Salud de la Republica de Colombia. Resolución No 008430 de 1993, en su titulo V sobre la Investigación Biomédica con animales. 1993. p. 11-12.
11. Osorio YS, Gonzalez J, Gama VL, Travi B. Reinfection in American cutaneous leishmaniasis: evaluation of clinical outcomes in the hamster model. Mem Inst Oswaldo Cruz. 1998;93:353-6.

12. Requena JM, Soto M, Doria MD, Alonso C. Immune and clinical parameters associated with Leishmania infantum infection in the golden hamster model. Vet Immunol Immunopathol. 2000;76:269-81.

13. Travi BL, Martinez JE, Zea A. Antimonial treatment of hamsters infected with Leishmania (Viannia) panamensis: assessment of parasitological cure with different therapeutic schedules. Trans R Soc Trop Med Hyg. 1993;87:567-9.

14. Travi BL, Osorio Y, Melby PC, Chandrasekar B, Arteaga L, Saravia NG. Gender is a major determinant of the clinical evolution and immune response in hamsters infected with Leishmania spp. Infect Immun. 2002;70:2288-96.

15. Van Hoosier GL, McPherson CW, Editors. The Laboratory Hamsters. Orlando: Academic Press; 1987. p. 10-398.

16. Varela-M RE, Muñoz DL, Robledo SM, Kolli BK, Dutta S, Chang KP, et al. Leishmania (Viannia) panamensis: an in vitro assay using the expression of GFP for screening of antileishmanial drug. J Exp Parasitol. 2009;122:134-9.

17. Wilson ME, Jeronimo SM, Pearson RD. Immunopathogenesis of infection with the visceralizing Leishmania species. Microb Pathog. 2005;38:147-60.

18. World Health Organization. Leishmaniasis: the global trend. Neglected tropical diseases. Geneva: WHO; 2009.

19. World Health Organization. Control of the leishmaniasis: report of a meeting of the WHO Expert Committee on the Control of Leishmaniases. WHO technical Report Series. Geneva: WHO; 2010.

20. Wyllie S, Fairlamb AH. Refinement of techniques for the propagation of Leishmania donovani in hamsters. Acta Trop. 2006;97:364-9.

Received: 25 September 2012

Accepted: 21 January 2013 